\title{
Mechanisms and therapies for acute CNS insults
}

\author{
Raghu Vemuganti $\cdot$ Heng Zhao
}

Published online: 6 July 2014

(C) Springer Science+Business Media New York 2014

Multiple synergistic mechanisms contribute to secondary brain damage following acute insults to CNS like stroke, traumatic brain injury (TBI) and spinal cord injury (SCI). The goal of this special issue is to discuss some of those pathways and the current experimental therapeutic approaches that help minimize the neurological dysfunction after acute CNS injuries.

Transcription factors are known to play a significant role in inflammation, oxidative stress and neuronal death as well as neuroprotection and plasticity after stroke. Yin et al. discuss the role of the zinc finger family of transcription factors; Krüppel-like factors in protecting neurons and capillaries after stroke. Cerebral inflammation is known to significantly influence neural progenitor cell proliferation. In this regard, Wang and Jin discuss the role of various inflammatory molecules in modulating neurogenesis after stroke. As GABA is the major inhibitory neurotransmitter in the mammalian brain and its dysfunction plays a role in secondary brain damage, $\mathrm{Wu}$ and Sun discuss the potential role of GABA in various forms of acute CNS injuries, including stroke and TBI.

Glial cells play important roles in protecting as well as exacerbating neuronal death after acute CNS insults by modulating inflammatory reactions. Kawabori and Yenari show the role of microglia in mediating secondary brain damage following stroke. Ziebell et al. discuss the potential of microglia in the neuronal damage afterTBI. Furthermore, Muradashvili et al. discuss the significance of

R. Vemuganti $(\bowtie)$

Department of Neurological Surgery, University of Wisconsin,

Madison, WI, USA

e-mail: vemuganti@neurosurgery.wisc.edu

H. Zhao

Department of Neurosurgery, Stanford University, Stanford, CA, USA matrix metalloproteinase-9 activation in mediating inflammation after TBI.

The significance of epigenetic modifications in secondary brain damage is poorly understood. In this regard, Kalani et al. discuss the therapeutic potential of the anti-inflammatory compound curcumin in modulating DNA methylation and histone modifications after stroke. Diabetes is a confounding promoter of stroke. Hence, Rehni et al. discuss the role of mitochondria in exacerbated post-stroke brain damage in the diabetic brain. Since, in certain cases, stroke can lead to intracerebral hemorrhage (ICH), Kathirvelu and Carmichael discuss the characteristics and therapeutic usefulness of various mouse models of ICH.

Despite testing over a thousand compounds in animal stroke models and over a hundred compounds in clinical trials, still, no drug that can prevent secondary brain damage and improve neurological outcome in humans has emerged. Jickling and Sharp discuss the roadblocks for therapeutic testing and the modalities to overcome them for finding novel stroke therapies. Furthermore, Cox et al. discuss the therapeutic potential of the drugs that target the pathophysiologic mechanisms after SCI.

Many physical therapies are being tested to control poststroke brain damage. In this regard, Wei et al. discuss the role of the galectin-9/Tim-3 signaling in modulating the antiinflammatory effects of ischemic post-conditioning, which is known to be neuroprotective, after stroke. Furthermore, Yu et al. discuss the therapeutic potential of another physical therapy modality, the near infrared light therapy, in preventing mitochondrial dysfunction after stroke. In addition, Venna and McCullough discuss the need for social support in functional and cognitive recovery after stroke.

Overall, this special issue is a compendium of the most recent understanding of the mechanisms and therapies for preventing secondary brain damage after acute CNS insults. 